S A Journal fo Industrial Engineering, Vol 7, No 7, December 1993, pp.12-23

\title{
THE CONCEPT OF AGE IN SYSTEMS ANALYSIS
}

\author{
A Dubi and N Gurvitz \\ Department of Nuclear Engineering \\ Ben-Gurion University of the Negev \\ Beer-Sheva \\ Israel \\ S J Claasen \\ Department of Industrial and Systems Engineering \\ University of Pretoria \\ Pretoria \\ South Africa
}

\section{ABSTRACT}

This paper discusses the mathematical concept of ageing. It is shown that while in most cases a probabilistic definition of age is sufficient, in some cases a calendaric definition must be added in order to preserve the relationship between time and age.

\section{OPSOMMING}

Hierdie artikel bespreek die wiskundige betekenis van veroudering. Daar word getoon dat alhoewel 'n waarskynlikheidsgebaseerde definisie van ouderdom meestal voldoende is, daar in sommige gevalle ' $n$ tydgebaseerde definisie nodig is om die verwantskap tussen tyd en ouderdom te behou. 


\section{INTRODUCTION}

The intuitive meaning of age is well known and commonly used in every aspect of life. Age usually refers to the time elapsed from the beginning of operation of an element (a birth time) to the current time. Age is therefore measured in time units appropriate to the element under consideration. Human life is measured in years, while the age of a rocket engine may be measured in seconds or minutes. The concept of age is also related to probability. By stating the age of an element one also gives some information on the probability that an event (a failure or any other type of change) will occur to the element in the near future. In order to have an idea about the probability of such an event, one must know the expected life of the element under consideration. For a human an age of 78 years implies a considerably higher probability of an event occuring within the next 5 years then at an age of 20 years. Terms such as young, middle aged and old refer to a range of ages which, (relative to the expected life time), gives information on the time elapsed since the birth time, and about the probability that a change will occur in a future time interval.

With the ability to model and analyse the behaviour of extremely complex systems, provided by general statistical simulation computer programs such as AMIR and SPAR $^{(1)}$, it becomes essential to introduce the concept of age of the system elements. Used in the context of the time behaviour analysis of systems, the concept of age must be uniquely and precisely defined. For example : What happens to the age of a system when a restoration operation is applied? Is it reset to zero, implying that the system becomes as good as new? What happens to the age of a system when it changes its mode of operation, for example from being in storage or being passive to being in an active state. All these questions are essential for realistic modeling. It is thus important to have a clear, unique and precise definition of the concept of age.

The purpose of this paper is to discuss the mathematical concept of ageing and to suggest a definition of this concept. 


\section{THE EXPONENTIAL CASE}

Let a component have a failure (or event) time distribution given by

$$
f(t)=\lambda e^{-\lambda t}
$$

$f(t)$ is the probability density function (Pdi) and $t$ is a random variable indicating the time of the event. Consider the situation shown in Figure 1. The component starts its life at $t_{o}$, it is currently at $t_{c}$, i.e. it reached the time point $t_{c}$ without any event occurring. The probability that a failure will occur between $t_{c}$ and $t_{f}$, which is some point in the future, is denoted by $P\left(t_{c}, t_{f} \mid t_{o}, t_{c}\right)$. It is the conditional probability for an event in $\left(t_{c}, t_{p}\right)$ given that no event happened in $\left(t_{b}, t_{c}\right)$ and can be expressed in general as:

$$
P\left(t_{c}, t_{f} \mid t_{o}, t_{c}\right)=\frac{F\left(t_{f}-t_{o}\right)-F\left(t_{c}-t_{o}\right)}{1-F\left(t_{c}-t_{o}\right)}
$$

where

$F(t)$ is the cumulative distribution of the failure time distribution.

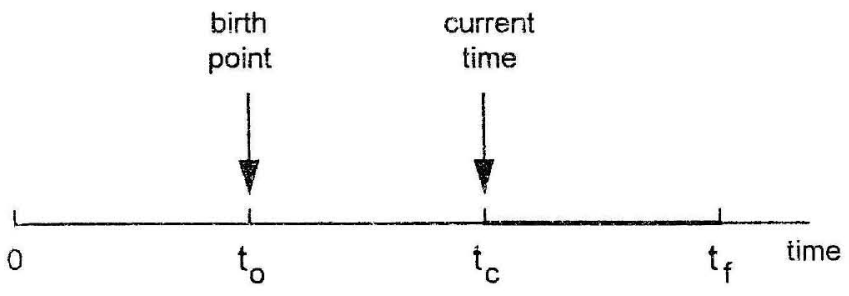

Figure 1. Time points in a component's life

Equation 1 is a good basis for a proper definition of age, as it describes the effect of the elapsed time since birth $\left(t_{c}-t_{o}\right)$, on the probability of an event occuring in the future. For the exponential distribution 
$F\left(t-t_{0}\right)=1-\lambda e^{-\lambda\left(t-t_{0}\right)}$

Substitution into equation (1) yields:

$P\left(t_{c}, t_{f} \mid t_{o}, t_{c}\right)=\frac{\lambda e^{-\lambda\left(t_{c}-t_{o}\right)}-\lambda e^{-\lambda\left(t_{f}-t_{o}\right)}}{\lambda e^{-\lambda\left(t_{c}-t_{o}\right)}}=e^{-\lambda\left(t_{f}-t_{c}\right)}$

The event probability for the exponential case is therefore independent of the elapsed time. It depends only on $\left(t_{f}-t_{c}\right)$, which is the future time interval under consideration. This result may be interpreted to indicate that the exponential distribution has no memory - the past has no effect on the future. One may say that every time point reached, is a birth point, or that the distribution is ageless. Thus a component is ageless with respect to an event governed by the exponential distribution and it is therefore appropriate to define the age under the exponential distribution as

$$
\text { Age } \equiv 0 \text {. }
$$

The exponential distribution is the only distribution that has this peculiar property. This can easily be shown by proving the inverse statement of equation (2) i.e. by proving that if $P\left(t_{c}, t_{f} \mid t_{o}, t_{c}\right)=g\left(t_{f}-t_{c}\right)$ (namely the conditional probability is a function of the future time interval only) then the governing cumulative probability density function ( Cdf), $F\left(t-t_{o}\right)$, must be an exponential distribution. This ageless property makes the mathematical handling of the distribution simple as one does not need to consider the past. This is, presumably, the reason why this distribution is so popular in reliability analysis and systems engineering. One should realize that when an exponential distribution is assumed, it implies that the component remains new at all times. Furthermore, even if the component fails, upon repair it is again new and remains new. Also note that the concept of preventive maintenarice 
becomes meaningless with the exponential distribution. Preventive maintenance is intended to renovate a component and thus to reduce its age - this is of course meaningless if the age is zero at ail times. Yet, any attempt to consider a distribution other than the exponential, makes the calculations (accept those of first event analysis or steady state properties of statistically independent components) prohibitively difficult. The calculation of the time dependent availability of a single component, with non-exponential distributions for either failure or repair, leads to an infinite convolution integrals series ${ }^{(2,3)}$. In this situation the Markov equations collapse (since they are only valid for systems with no memory i.e. Markovian systems) and instead a simultaneous set of voltera type integral equations $s^{(4)}$ hold.

In reality nothing is ageless. All entities age - a wallet, a cigarette lighter, a television set, a telephone, even planets and the universe as a whole go through physical processes of aging. Thus, modeling reality in terms of the exponential distribution is often unfounded. Still one may argue that over certain life time intervals an exponential approximation is justified. This argument combined with the difficulty in handling "aging" distributions and the lack of data (and one may wonder to what extent the desire to obtain exponential distributions, influences the data collection process) makes the exponential distribution a "default" choice.

However, the emergence of multipurpose simulation tools as well as other stochastic simulation methods, make the handling of non-exponential distributions a feasible engineering option. Thus the consideration and definition of age becomes relevant.

\section{DEFINITION OF AGE}

Since age is measured in units of time, it would seem that time elapsed since birth, would serve as an appropriate measure of age. However this unit of measure, suffers from two serious deficiencies which makes it undesirable. Firstly, age may range to infinity and thus involves large numbers which may be inconvenient to handle and secondly, and more importantly, such a definition of age would not yield an idea about the actual state of a component. If, for example, the age of a component is stated as 200 hours it is not clear whether the component is young or old. If the mean life of 
the component is 130 hours then 200 hours is a rather old age, yet if the mean life is $2 \times 10^{6}$ hours the component is very young. Thus age, measured in time units elapsed since birth, does not indicate the true age of the component under consideration. What is needed is a definition of age that gives information on the probability state of the component and will have the same applicability independent of the specific distribution which governs the component's behaviour. A definition that meets this demand and does not suffer from the deficiencies described above is

$$
\operatorname{Age}(t)=F\left(t-t_{o}\right)
$$

This is simply the Cdf of the failure (or event) time distribution for the component. It is a monotonic increasing function and its range is between 0 and 1. If the age is, for example $0.02,0.5$ or 0.99 it means that the component is very young, in mid life or very old respectively. Thus it yields direct information as to the state of life of the component, independent of the specific age distribution. An age of 0.32 means that the component traversed a time interval, in which it had a probability of 0.32 to fail, without failure. Note also that $P\left(t_{c}, t_{f} \mid t_{o}, t_{c}\right)$ takes the form

$$
P\left(t_{c}, t_{f} \mid t_{o}, t_{c}\right)=\frac{\operatorname{Age}\left(t_{f}\right)-\operatorname{Age}\left(t_{c}\right)}{1-\operatorname{Age}\left(t_{c}\right)}
$$

The hazard function can aiso be expressed in this form

$$
z\left(t_{c}-t_{o}\right)=\frac{f\left(t_{c}-t_{o}\right)}{1-F\left(t_{c}-t_{o}\right)}=\lim _{\substack{t_{f} \rightarrow t_{c} \\ t_{f}>t_{c}}} \frac{\operatorname{Age}\left(t_{f}\right)-\operatorname{Age}\left(t_{c}\right)}{\left(t_{f}-t_{c}\right)\left(1-\operatorname{Age}\left(t_{c}\right)\right)}
$$


event probability. Let the age of a component be described by the cumulative distribution of a Weibull distribution of the form

$$
F\left(t-t_{0}\right)=1-e^{-\lambda\left(t-t_{0} t^{t}\right.}
$$

with $\lambda=3 \cdot\left(10^{-5}\right), K=1.3$ and $t_{o}=0$

At $t=1600$ hours the age of the component is 0.3552 , calculated as follows:

$$
\operatorname{Age}(1600)=1-e^{-\lambda \cdot(1600)^{13}}=0.3552
$$

The probability that a failure will occur in the 200 hours between 1600 and 1800 is (using equation (1)):

$$
P_{1}=\frac{F(1800)-0.3552}{1-0.3552}=0.07025
$$

As time progresses to 3000 hours the age of the component reaches 0.6299 . The probability for an event in the next 200 hours is now:

$$
P_{2}=\frac{F(3200)-0.6299}{1-0.6299}=0.08331
$$

The mean life of the above distribution is 2773.4 hours. At 5400 hours the age reaches 0.8816 and the probability for an event in the next 200 hours is 0.0986 . Thus it is seen that the age increase is much faster than the increase in the probability of the future event for a given time interval. It should also be noted that for any given distribution the age and the time uniquely determines each other. Knowing the probabilistic age the time unit age can be found by solving the inverse of equation (3). 


\section{AGE CONSERVATION RULES}

Consider a component or a system which changes its operating conditions, such as a car changing from being parked to being in motion, from motion into high speed motion or into motion on a difficult road or even from city traffic into open road conditions. Each different operational state will be referred to as a different profile state of the system. Most systems are operating with at least two profile states such as operation and rest. In each profile state the component is governed by a different pdf. Assume that the component is in state 1 up to time $t_{p}$ at which the profile state changes into state 2 . In state 1 the failure (or event) distribution is $f_{1}\left(t-t_{o}\right)$ and in state 2 it is $f_{2}\left(t-t_{o}^{\prime}\right)$. At point $t_{p}$ the age of the component is Age $\left(t_{p}\right)=F_{1}\left(t_{p}-t_{o}\right)$. What happens to the age as the component moves to the second profile state? How should one calculate the future event probability $P\left(t_{p}, t_{f} \mid t_{o}, t_{p}\right)$ in this case? Although this is an interesting mathematical question its importance lies within the realm of reality. Since this situation is part of the everyday reality of many systems, the above questions are relevant to the way in which one models this reality. It is to be noted that $f_{2}\left(t-t_{0}^{\prime}\right)$, contains the birth point $t_{o}^{\prime}$, as an undefined parameter since the component did not start its life at any point with this distribution. A reasonable modeling assumption would be a conservation rule stating that the component retains the age accumulated up to $t_{p}$ and proceeds into the future from that point on. This assumption is supported by the argument that just by changing the governing distribution the system does not suddenly get "older" or "younger". The age conservation assumption suggests that at $t_{p}$

$$
F_{2}\left(t_{p}-t_{o}^{\prime}\right)=\operatorname{Age}\left(t_{p}\right)=F_{1}\left(t_{p}-t_{o}\right)
$$


hence $t_{o}^{\prime}$ is set as

$$
t_{o}^{\prime}=t_{p}-F_{2}^{-1}\left[\operatorname{Age}\left(t_{p}\right)\right]
$$

This implies that in profile state 2 the situation is viewed as if the system follows the distribution $f_{2}\left(t-t_{o}^{\prime}\right)$ with a birth point that is determied by the age accumulated up to $t_{\mathrm{p}}$. Note that $t_{o}^{\prime}$ may be negative. The probability for a future event is then given by

$$
P=\frac{F_{2}\left(t-t_{o}^{\prime}\right)-\operatorname{Age}\left(t_{p}\right)}{1-\operatorname{Age}\left(t_{p}\right)}
$$

where $t_{o}^{\prime}$ is defined by equation (7).

An interesting problem arises when the profile change leads the system from a nonexponential distribution into a state governed by an exponential distribution. Noting that the exponential distribution has no memory and that the age imposed under it is zero, one may wonder what should be the age or, what happens to the age accumulated in the first profile state. Obviously, equations (6), (7) and (8) are meaningless for the exponential distribution, and independent of the age reached, equation (2) applies, which means that if we transfer to an exponential distribution the age is reset to zero. If the distribution reverts back to a non-exponential distribution (see fig 2), with what age does the system start at $t_{2}$. It can be either a zero age or the age reached at $t_{1}$. Both possibilities are mathematically consistent and at $t_{2}$ equations (6) to (8) apply. The choice between zero age or preserved age only depends on the real situation being modelled. If the system is a "as good as new" upon reaching $t_{2}$ then the age at $t_{2}$ is zero, otherwise the age at $t_{1}$ is preserved. If the age increases that from $t_{1}$ to $t_{2}$ then the distribution between $t_{1}$ and $t_{2}$ cannot be exponential. 


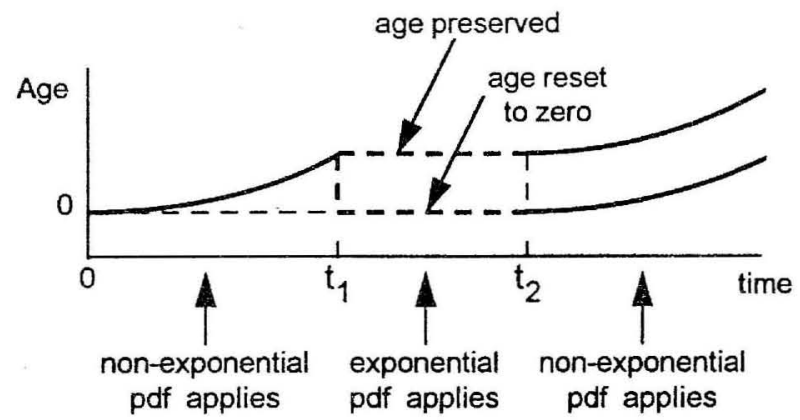

Figure 2: Flipping from non-exponential to exponential distribution and back

\section{NON-PARAMETRIC DISTRIBUTIONS}

In many cases the data collection process results in a piecewise constant pdf rather than a continuous pdf. Thus $f(t)$ is constant over a set of time intervals (see figure 3 ). This implies that the probability that the event

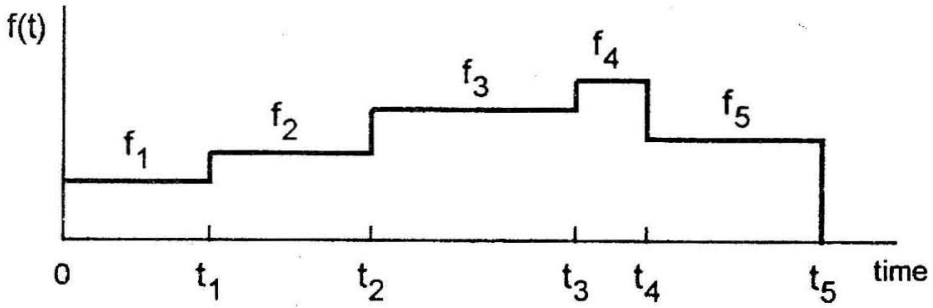

Figure 3 : Non-parametric distribution

will occur in the $i^{\prime}$ th interval is $P_{i}=f_{i}\left(t_{i}-t_{i-1}\right)$ and the distribution within each interval is uniform. The definition of equation (3) would still apply, taking the form

$$
\operatorname{Age}(t)=F(t)=\sum_{k=0}^{i-1} f_{k}\left(t_{k+1}-t_{k}\right)+f_{i}\left(t-t_{i-1}\right) \text { for } t_{i-1} \checkmark t \leq t_{i}
$$

However, an interesting problem arises if the Pdf has a zero value in one or more intervals (note that this can never be the last interval). 
Consider the situation in which a repair event of a system may take up to 120 hours. The first 100 hours are used for various preliminary activities (diagnostics, dismantling, documentation etc) such that the repair is never completed before 100 hours. Following the 100 hours the repair may be completed uniformly at any point between 100 and 120 hours. The Pdf of such a repair event is adequately modelled by a piecewise constant Pdf being zero in $(0,100)$ and $\frac{1}{20}$ in $(100,120)$ as illustrated in Figure 4.

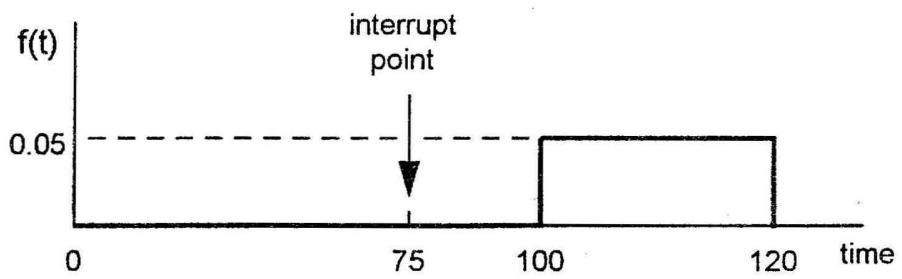

Figure 4 : Zero interval repair pdf

The probability definition of age implies that within the first interval the age remains zero. What is the "reality" interpretation of such a situation? Does it mean that while time is progressing the "age" is "frozen"? Consider the possibility that in the above example the repair process is interrupted at $(t+75)$ for 72 hours and then continued. Where does it continue from? Since the age is zero it may continue at any point in the first interval. The one to one relation of time as function of age is lost i.e. the probabilistic age is not sufficient to define the time unit age. Obviously, the restart point for the repair, in the above example, depends on the "reality" of the situation. If during the interrupt period all the information about the repair is lost, then the repair restarts again at the beginning. If, on the other hand, the repair constitutes a set of ordered preliminery operations and the information concerning them is not lost, then the repair should restart at the interrupt point.

Since the probabilistic definition of age does not preserve the information about elapsed time in the case of zero Pdf intervals, a calendaric definition of age seems appropriate for piecewize constant Pdf's with zero intervals. Such a definition would 
be:

$$
\operatorname{Age}_{c}(t)=\frac{t}{T_{\max }}
$$

where $T_{\max }$ is the domain of the distribution. Since non-parametric distributions have a finite domain this definition is also defined in the range $[0,1]$ and preserves the one to one relation between time and age. Note that from the calendaric age one can always obtain the probabilistic age but not vice versa. Also in the calculation of the future event probability (equation (8)) the probabilistic age must be used.

\section{CONCLUSION}

The concept of "age" plays a significant role in the modeling of systems characterised by nonexponential distributions. The considerations involved in the definition and relation of age and time were discussed. It was shown that whereas in most cases a probabilistic definition of age is appropriate, in some cases a calendaric definition must be added to preserve the relation between time and age. These definitions of age are extensively used in the general multi purpose stochastic simulation codes, AMIR and SPAR, to analyse the time dependent performance and behavior of complex realistic systems.

\section{REFERENCES}

1. Dubi A., "Stochastic Modeling of Realistic Systems", Tutorial notes, $\mathbf{R} \& \mathbf{M}$ Simposium, Los Angeles, January 1990.

2. Barlow R.E. and Proschan F., Mathematical Theory of Reliability, John Wiley \& Sons, 1965.

3. Cleroux R. and McConalogue D.J., "A Numerical Algorithm for Recursively-Defined Convolution Integrals involving Distribution Functions", Management Science, pp. 1138, 1976.

4. Dubi A., "Integrated Problems Involved in Spare Parts Allocation and Maintenance in Realistic Industrial Systems", Workshop - SOLE Conference, South Africa, 1991. 